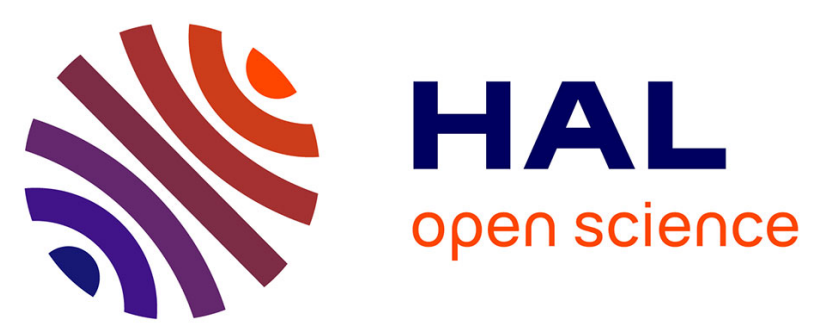

\title{
Monte Carlo Modeling of Electron Multiplication in Amorphous Silicon Based Microchannel Plates
}

Janina Löffler, Jonathan Thomet, Mohamed Belhaj, Luc Van Kessel, Cornelis W Hagen, Christophe Ballif, Nicolas Wyrsch

\section{- To cite this version:}

Janina Löffler, Jonathan Thomet, Mohamed Belhaj, Luc Van Kessel, Cornelis W Hagen, et al.. Monte Carlo Modeling of Electron Multiplication in Amorphous Silicon Based Microchannel Plates. 2019 IEEE Nuclear Science Symposium and Medical Imaging Conference (NSS/MIC), Oct 2019, Manchester, United Kingdom. 10.1109/NSS/MIC42101.2019.9059971 . hal-02971313

\section{HAL Id: hal-02971313 \\ https://hal.science/hal-02971313}

Submitted on 19 Oct 2020

HAL is a multi-disciplinary open access archive for the deposit and dissemination of scientific research documents, whether they are published or not. The documents may come from teaching and research institutions in France or abroad, or from public or private research centers.
L'archive ouverte pluridisciplinaire HAL, est destinée au dépôt et à la diffusion de documents scientifiques de niveau recherche, publiés ou non, émanant des établissements d'enseignement et de recherche français ou étrangers, des laboratoires publics ou privés. 


\title{
Monte Carlo Modeling of Electron Multiplication in Amorphous Silicon Based Microchannel Plates
}

\author{
J. Löffler, J. Thomet, M. Belhaj, L. van Kessel, C. W. Hagen, C. Ballif, N. Wyrsch
}

\begin{abstract}
Amorphous silicon based microchannel plates are being developed to overcome performance limits of conventional microchannel plates. They offer a new flexibility and ease of fabrication. A comprehensive AMCP model is being constructed to analyze the performances of AMCPs. It includes Monte Carlo simulation of secondary electron emission distribution as a function of energy and angles and finite element analysis multiphysics software to compute electron trajectories. The paper presents the results of Monte Carlo simulations of secondary emission functions in silicon and the high secondary emissive material $\mathrm{Al}_{2} \mathrm{O}_{3}$. We discuss the gain and potential performance as a function of geometry of such devices. The validity of the Eberhardt model for the analysis of AMCPs is also addressed.
\end{abstract}

\section{INTRODUCTION}

Developments in nanofabrication have enabled a new generation of microchannel plates based on amorphous silicon (AMCPs) [1,2]. Like in a conventional microchannel plate, incoming electrons are accelerated in the electric field of the channels. Every time an electron hits the channel wall, secondary electrons are created. Passing through the channel, an electron is multiplied by a device specific factor, its gain. There are two different theoretical approaches to describe the multiplication process in MCPs. Historically, the first model was assuming discrete dynodes [3,4]. It was recently further developed into a transmission line model [5]. The transmission line models were developed to describe MCPs under irradiation with an electron pulse. The second approach is to follow the trajectory of every electron with Monte Carlo modeling. Monte Carlo models for MCPs have been developed since 1971 [6], and are used to simulate MCPs for single photon detector applications, to calculate the gain, deduce timing properties [7] and the influence of different coatings on the signal to noise ratio [8].

J. Löffler, J. Thomet, C. Ballif and N. Wyrsch are with the Photovoltaics and Thin-Film Electronics Laboratory, Institute of Microengineering (IMT), Ecole Polytechnique Fédérale de Lausanne (EPFL), Neuchâtel, Switzerland (e-mail first author: janina.loffler@epfl.ch).

M. Belhaj is with ONERA, The French Aerospace Lab, 31055 CEDEX, France.

L. van Kessel and C.W. Hagen are with Delft University of Technology, Dept. ImPhys, Lorentzweg 1, 2628 CJ Delft, The Netherlands.

\section{MODELING ELECTRON TRAJECTORIES}

Electron multiplication in microchannel plates is driven by two factors: the electric field and the secondary emission coefficient. A schematic drawing of an AMCP and the interaction at a channel wall are shown in Fig. 1. We use a finite element method to model the electric field of AMCPs depending on the channel geometry. The goal of this model is to analyze AMCP behavior for high aspect ratio AMCPs currently being developed. More generally, it enables us to investigate the effect of various channel geometries on AMCP gain and timing properties. We are modeling the secondary emission based on a Monte Carlo approach as described in [9]. At each interaction of an electron with the channel wall, secondary electrons are emitted according to a probability that depends on the energy and the impact angle of the incoming electron. The number of emitted electrons, their emission angles and energies are drawn from probability distributions. These probability distributions strongly depend on the incoming electron energy and its angle.

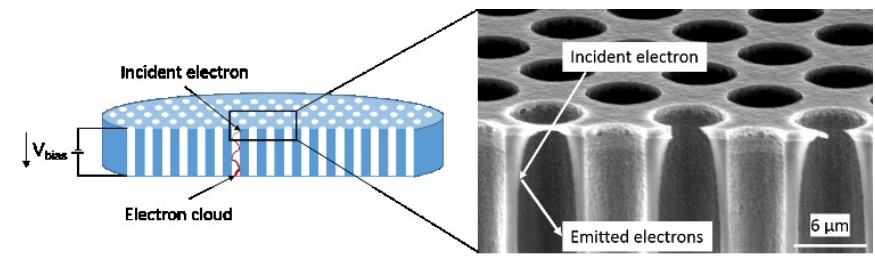

Fig. 1. Schematic drawing of amorphous silicon based microchannel plate and emission of electrons from an electron interacting with the channel wall, creating an electron cloud.

Energies of secondary electrons are generally low, normally defined as electrons with energies below $50 \mathrm{eV}$. For a standard AMCP sample in standard operating conditions, a maximum outgoing energy of up to $40 \mathrm{eV}$ for electrons exiting the channel was measured [10]. This means the average energy of electrons hitting the channel wall will also be in the low energy range. Both, measurements and calculations of the secondary yield in this energy range are challenging and not known from literature. Measurements are very sensitive to the surface composition and roughness. For the simulation, we are using a Monte Carlo model for electron scattering. Measurements and model results are fitted for all incoming energies and angles. The relative yield for different angles of incidence is determined from the electron scattering model.

Measurements of the energy distribution of the emitted electrons let us determine the secondary emission probability 
and the probability density function for the electron energy of the outgoing electron. The angular distribution of the emitted secondary electrons is assumed to be a cosine distribution in the polar direction and isotropic in azimuthal direction [11].

\section{BACKSCATTERED VS. EMITTED ELECTRONS}

Measurements of electron emission energies and electron yield were done at the electron emission analysis facility at ONERA Space Lab, described here [12,13]. The emitted energy distribution was measured on a thin layer of amorphous silicon (a-Si:H) of $17 \mathrm{~nm}$ thickness, on a Si wafer. Fig. 2 shows the energy spectra for different incoming electron energies that were collected using the hemispherical electron energy analyzer. The spectra have been normalized to compare the different incoming energies of $13.5 \mathrm{eV}, 43.5 \mathrm{eV}$, $93.5 \mathrm{eV}$ and $193.5 \mathrm{eV}$.

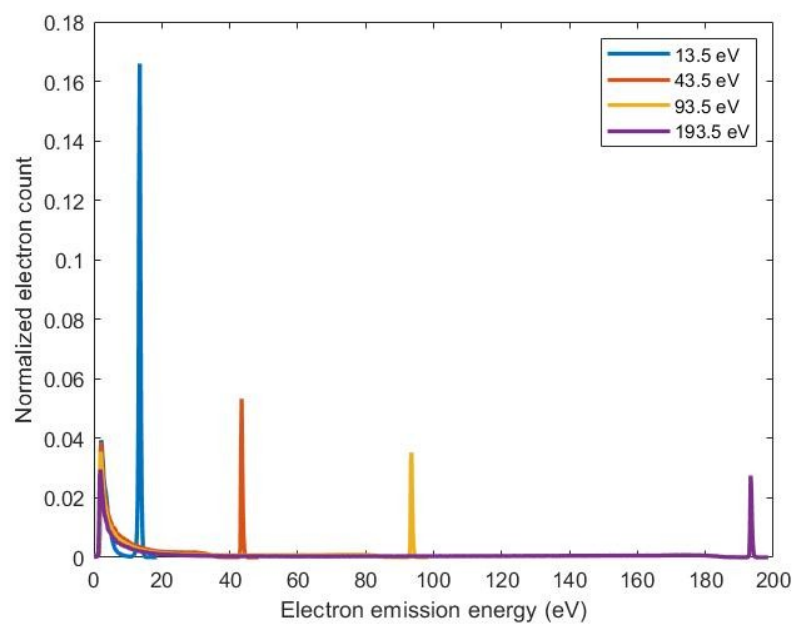

Fig. 2. Normalized electron emission spectra of a-Si:H measured at $45^{\circ}$ incidence for incoming electron energies of $13.5 \mathrm{eV}, 43.5 \mathrm{eV}, 93.5 \mathrm{eV}$ and $193.5 \mathrm{eV}$. The backscattering probability is higher for lower incoming electron energies.

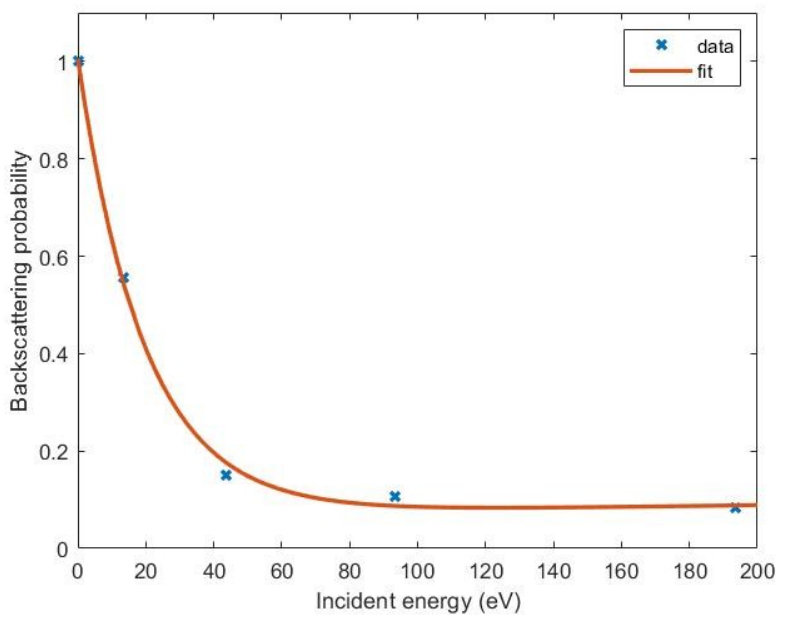

Fig. 3. Backscattering probability of a-Si:H depending on the incoming energy. As the number of secondary electrons created tends towards 0 for energies closer to 0 , the backscattering probability for $0 \mathrm{eV}$ has been defined as 1 .

The fraction of backscattered electrons becomes smaller with higher electron energies. For our purpose of AMCP modeling, we separate electrons in 1) elastically backscattered electrons and 2) primary and secondary electrons that are (re-) emitted from the material. This distinction is made because elastically backscattered electrons reverse their direction whereas the emission angles for electrons emitted from the material follow a cosine distribution [14]. As we are looking at low energies here, the conventional definition of secondary energies being all energies below $50 \mathrm{eV}$ [15] is clearly not valid. We define the backscattered electrons as the electrons with energies similar to the energy of the incoming electron $E_{\text {in }}$. The number of backscattered electrons $N_{B S}$ is then

where $N(E)$ is the number of electrons per emission energy. We define emitted electrons accordingly as all electrons with lower energies than $E_{\text {in }}-1.5 \mathrm{eV}$ and their number $N_{E E}$ as

As the amount of inelastically backscattered electrons is low at low energies, most of the emission stems from secondary electrons. We calculated the backscattering probability

Fig. 3 shows the data points and the fit function for the backscattering probability

, $c$ and $d$ are fit parameters, and the function was chosen to fit the data points. The parameter values for all fits in this paper can be found in the appendix. We added the backscattering probability of $P_{B S}(0 \mathrm{eV})=1$, as the secondary yield tends to 0 as the electron energy tends towards $0 \mathrm{eV}$ [16]. At these energies, electrons are either backscattered or absorbed into the material. Using the fit function, the backscattering probability can easily be determined at each impact, depending on the incident energy.

\section{ELECTRON EMISSION YIELD}

The total emission yield of the a-Si:H layer and of a $8 \mathrm{~nm}$ $\mathrm{AlO}_{\mathrm{x}}$ layer on $\mathrm{Si}$ has been measured with the method described in [12]. The electron yield measurements are shown in Fig. 4. The yield was measured for normal incidence, defined as $0^{\circ}$ incidence. A coating of the channel walls with the alumina layer clearly improves the electron yield of AMCPs.

In AMCPs, electrons are accelerated in the electric field of the channels. When they reach a channel wall, the incident angle is significantly higher than $0^{\circ}$ (with a maximum of almost $90^{\circ}$ ). For this reason, we used an electron scattering model in addition to the measurements to determine the electron yield at different incident angles. The electron scattering simulations are similar to the model described by Mao et al [17], with modifications to include inner-shell ionization [18,19] and electron-phonon interaction below 100 
$\mathrm{eV}$ [18-20]. The calculated total electron yield of $\mathrm{Si}$ and $\mathrm{Al}_{2} \mathrm{O}_{3}$ are shown in Fig. 5 for incident angles of $0^{\circ}$ and $80^{\circ}$ and energies up to $500 \mathrm{eV}$. We use the calculated total electron yield curves of silicon to extrapolate the angular dependence of the electron emission yield of a-Si:H. The same parametrization can be done for $\mathrm{AlO}_{\mathrm{x}}$ using the backscattering probability of energy emission spectra of the $\mathrm{AlO}_{\mathrm{x}}$ sample.

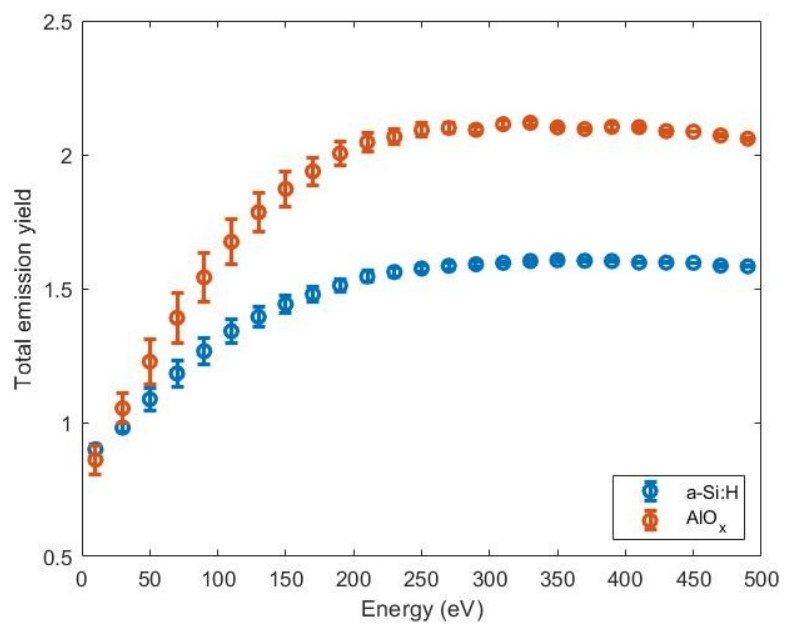

Fig. 4. Experimental electron emission yield, measured at $0^{\circ}$ incidence at the ONERA Space Lab.

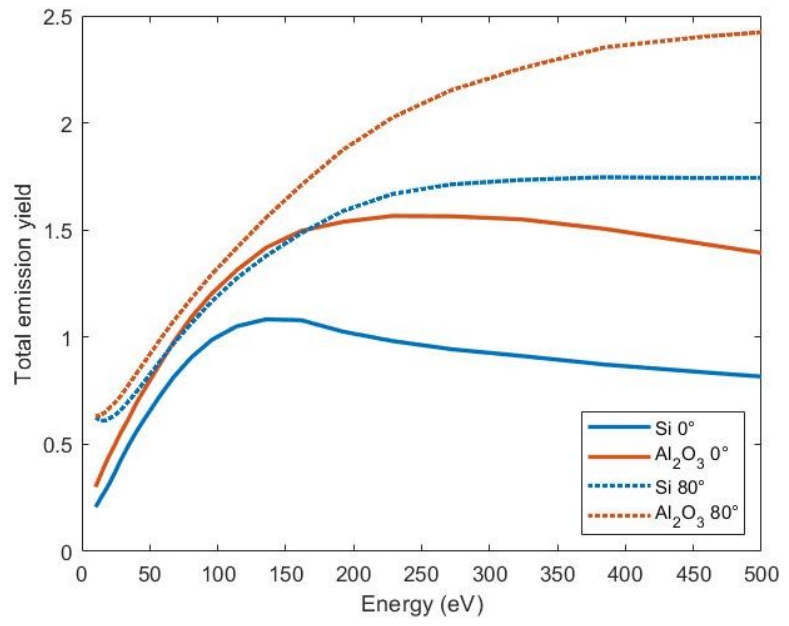

Fig. 5. Simulated electron emission yield of $\mathrm{Si}$ and $\mathrm{Al}_{2} \mathrm{O}_{3}$ at angles of incidence of $0^{\circ}$ and $80^{\circ}$.

We parameterize the electron yield curves according to [21] and [9]. The maximum yield at $0^{\circ}$ is $\delta_{\max }=1.08$ at an energy of $E_{\max }=135.6 \mathrm{eV}$. With this, we fit the following equation:

with . From here, the yield at higher angles of incidence is calculated using the formula
The fits for angles of $0^{\circ}, 30^{\circ}$ and $60^{\circ}$ are shown in Fig. 6. As the graph shows, the model describes the calculated yields very well.

The measured electron yield of a-Si:H was fitted to a modified model from [21], adding a constant fit parameter $a$ for the backscattered yield:

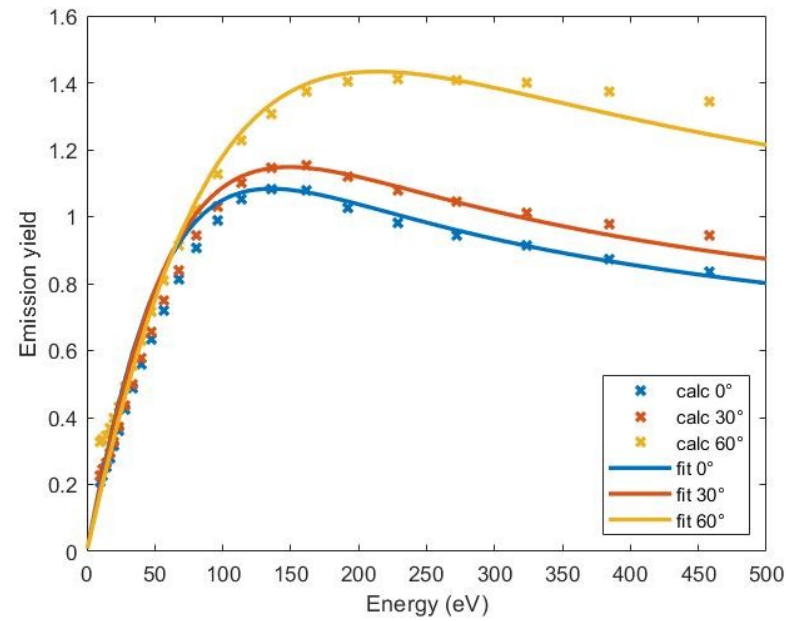

Fig. 6. Fitted electron emission yield for simulated data at $0^{\circ}$. The emission yield at higher angles has been deduced from the fit at $0^{\circ}$ with the formula given in equation (6).

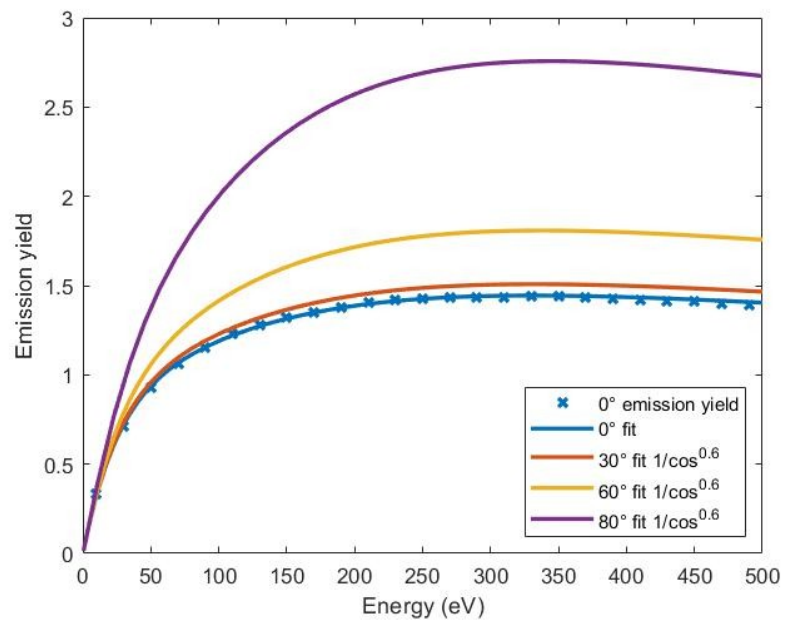

Fig.7. Fitted electron yield curve with different angular dependencies to fit the electron yield curves at higher angles of incidence. For the model a value of - was chosen, from the results of the calculated yield curves. The backscattered electrons have been removed according to equation (4).

The yield curve at higher angles was then approximated with the factor $-x$, where $x$ should be deduced from measurements at higher angles of incidence. Fig.7. shows the fits with this model for $0^{\circ}$ incidence and also at higher incidence angles, assuming a behavior similar to the calculated yield. Additionally, we removed backscattered electrons according to equation (4). 
Thus, we chose the following dependencies to model the emission yield at higher angles:

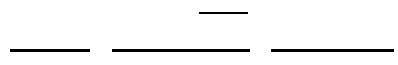

From the yield value, the actual number of emitted electrons is chosen from a Poisson distribution with

The expected value $\delta$ is the electron yield, and we fixed the maximum number of emitted electrons to 10 .

The overall yield, even at an angle of $80^{\circ}$ is lower than expected from AMCP measurements that were fitted to the Eberhardt model with a value of $\delta=1.7$ [2]. This can be due to the surface morphology in AMCP channels that could be beneficial for secondary emission. As the channels are etched using a Bosch process, the channels are not perfectly straight and show scalloping.

\section{EMISSION ENERGY DISTRIBUTION}

The emission energies of the secondary and inelastically backscattered electrons follow the distributions shown in Fig. 2 and depend on the incoming electron energy. As the emitted electron energies lie mostly between $0 \mathrm{eV}$ and $40 \mathrm{eV}$, the emission spectrum for an incoming electron with $43.5 \mathrm{eV}$ is used for the probability distribution of emitted electrons. For a number of secondary electrons $\mathrm{N}$, the condition $\sum$

has to be met, where $E_{n}$ is the energy of the emitted electrons $1 \ldots \mathrm{N}$, and $E_{\text {in }}$ is the energy of the incoming electron. The probability density function used for the emission energy and the corresponding cumulative distribution function are shown in Fig. 8.

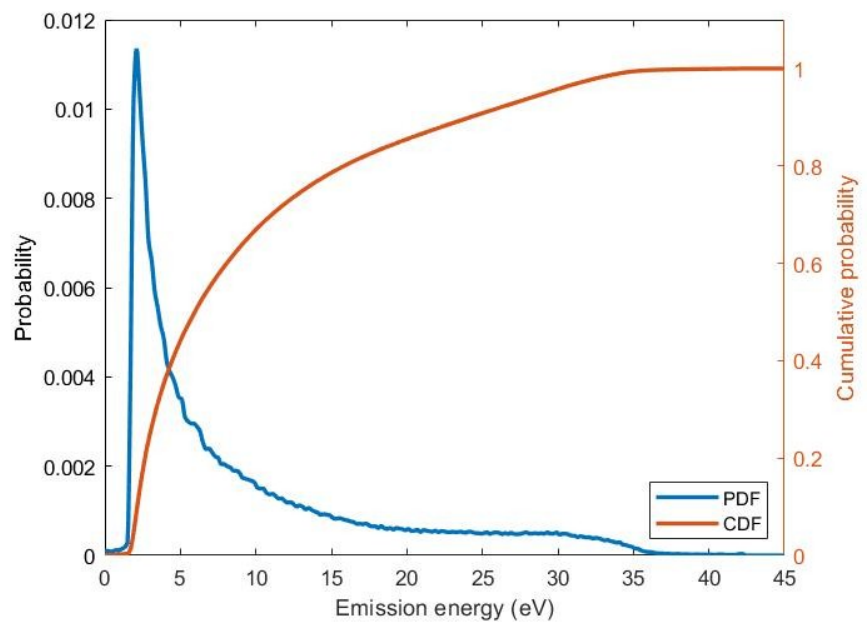

Fig. 8. Probability density function (PDF) and cumulative distribution function (CDF) for the emission energy of a secondary electron. The PDF is drawn from the emission spectrum at $43.5 \mathrm{eV}$ incidence as this corresponds to the energy of electrons in the channel.

\section{ELECTRON TRAJECTORIES}

We calculated electron trajectories in a 2D COMSOL model for one microchannel. Using the angular distribution, we show the angular and energy spread of electrons emitted from one point in the channel, shown in Fig. 9. The geometry of the microchannel corresponds to an AMCP sample with a channel diameter of $6.6 \mu \mathrm{m}$. The emission point is located at $(0,0)$ and the channel entrance placed $5 \mu \mathrm{m}$ above. The total channel length is $89.9 \mu \mathrm{m}$. The results shown here are calculated for an electric field of $5.5 \times 10^{6} \mathrm{~V} / \mathrm{m}$. The emission angle was randomly chosen from a cosine distribution. In Fig. 9. we used the energy emission spectrum shown in Fig. 8. To evaluate the impact angle distribution, we chose two energy values from the energy emission spectrum.

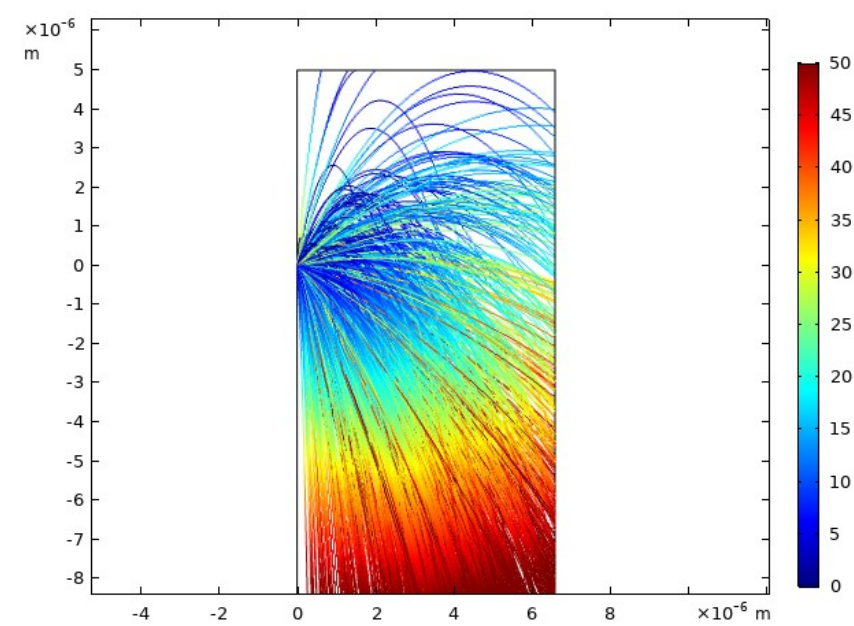

Fig. 9. Electron trajectories of 1000 electrons in a 2D microchannel, simulated with the finite element software COMSOL. The electron energies are random values from the emission spectrum of Fig. 8 and their emission angles are random values from a cosine distribution. The color chart shows the electron energy in $\mathrm{eV}$.

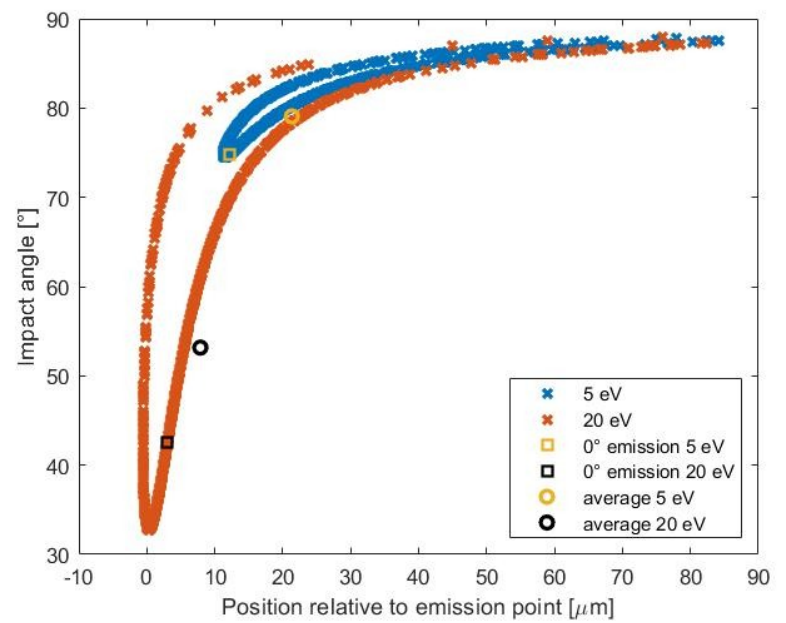

Fig. 10. Electron impact point and angular spread depending on the emission energy of electrons of $5 \mathrm{eV}$ and $20 \mathrm{eV}$.

Fig. 10 shows the distribution of the impact angle for the two emission energies, $5 \mathrm{eV}$ and $20 \mathrm{eV}$ and the position relative to the emission point. The impact positions and angles are widely spread for both energies. Fig. 11 shows a 2D simulation for emitted electrons with energies between $1 \mathrm{eV}$ and $50 \mathrm{eV}$, emitted normal to the surface. The average impact energy is higher for a lower emission energy and vice versa. In 
general, for a wide range of emission energies between $7 \mathrm{eV}$ and $50 \mathrm{eV}$, the impact energy is around $50 \mathrm{eV}$ and the distance traveled under $10 \mu \mathrm{m}$. This shows that there is an average of impact energies and distance travelled in the channel, which is the basic assumption of the Eberhardt model. However, the trajectories in a $2 \mathrm{D}$ channel, merely represent a maximum for trajectories in the 3D channel. In the $3 \mathrm{D}$ case, the azimuthal emission is random and for a fixed energy and polar angle, so the impact location can take all values up to the maximum shown in Fig. 11.

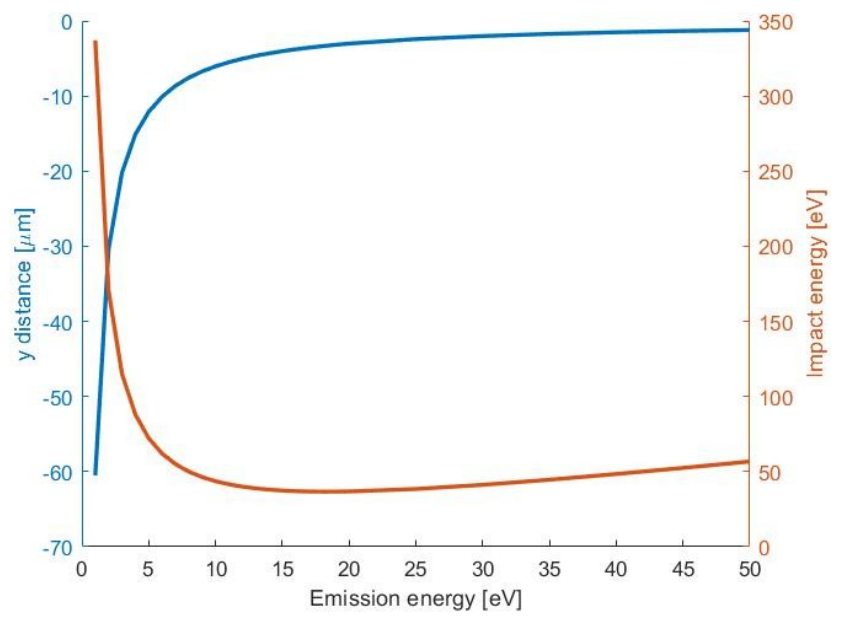

Fig. 11. 2D simulation of electrons emitted normal to the surface in a microchannel with emission energies from 0-50 eV. At very low energies the electrons travel up to several tens of $\mu \mathrm{m}$ and arrive with a high impact energy. Higher energy electrons above $5 \mathrm{eV}$ arrive with an energy similar to their emission energy and travel under $20 \mu \mathrm{m}$.

As a next step, the secondary yield and the energy emission distributions will be implemented in a 3D model to predict gain and timing of AMCPs for different geometries.

\section{CONCLUSION}

Electron trajectories in AMCPs can be modeled using emission statistics from measured and calculated data. The results shown here set the basis to gain a complete understanding of the benefits and the limits of this modeling approach. Additionally, the electron yield at low energies below $50 \mathrm{eV}$ has to be measured at different angles of incidence for a comprehensive model, as this plays an important role in determining the gain of a microchannel. Simulations at low energies show the angular dependence of the electron yield, although the absolute yield value is underestimated. As gain values of AMCPs up to 100 have been measured $[1,10]$, we expect the average emission yield of low energy electrons inside the channel to be higher than 1 . Implementing these results in a 3D model of a microchannel will let us compare the approach presented here with measurements and the gain model of Eberhardt. Furthermore, we will be able to calculate the AMCP gain and timing properties, e.g. the transit time and the timing jitter, for various channel geometries.

\section{Appendix}

\section{TABLE 1. FIT PARAMETERS}

$\begin{array}{ll}\text { Backscattering } & \text { probability equation (4): } \\ \text { Parameter } & \text { Value } \\ \text { a } & 0.9338 \\ \text { b } & -0.05092[1 / \mathrm{eV}] \\ \text { c } & 0.07154 \\ \text { d } & 0.001085[1 / \mathrm{eV}]\end{array}$

Maximum yield at $0^{\circ}$ equation (5):

$\begin{array}{ll}\text { Parameter } & \text { Value } \\ \mathrm{x} & 2.4539 \\ \mathrm{n} & 1.3\end{array}$

Measured yield at $0^{\circ}$ equation (7):

$\begin{array}{ll}\text { Parameter } & \text { Value } \\ \mathrm{a} & 0.8229 \\ \mathrm{~b} & 0.08589 \\ \varepsilon & 80 \\ \lambda & 40 \\ \mathrm{n} & 1.209\end{array}$

\section{ACKNOWLEDGMENTS}

We thank Dr. Nenad Bundaleski for his support and for sharing his insights on the angular dependence of the secondary electron yield. We are thankful to our colleague Samira Frey for preparing the a-Si:H sample and we also thank Dr. Juan J. Diaz Leon from the Swiss Center for Electronics and Microtechnology (CSEM) for preparing the alumina sample.

\section{REFERENCES}

[1] A. Franco, J. Geissbühler, N. Wyrsch, and C. Ballif, "Fabrication and characterization of monolithically integrated microchannel plates based on amorphous silicon," Sci. Rep., vol. 4, no. August 2015, pp. 1-7, 2014.

[2] J. Löffler, C. Ballif, and N. Wyrsch, "Amorphous silicon-based micro-channel plate detectors with high multiplication gain," Nucl. Instruments Methods Phys. Res. Sect. A Accel. Spectrometers, Detect. Assoc. Equip., 2018.

[3] E. H. Eberhardt, "Gain model for microchannel plates," Appl. Opt., vol. 18, no. 9, pp. 1418-1423, 1979.

[4] E. H. Eberhardt, "An Operational Model for Microchannel Plate Devices," IEEE Trans. Nucl. Sci., vol. 28, no. 1, pp. 712-717, 1981.

[5] L. Giudicotti and R. Pasqualotto, "Characterization of fast microchannel plate photomultipliers for the ITER core LIDAR Thomson scattering system," J. Instrum., vol. 7, no. 02, pp. C02037-C02037, 2012.

[6] A. J. Guest, "A computer model of channel multiplier plate performance," Acta Electron., vol. 14, no. 1, pp. 79-97, 1971.

[7] C. A. Kruschwitz, M. Wu, and G. A. Rochau, "Monte Carlo simulations of microchannel plate detectors. II. Pulsed voltage results," Rev. Sci. Instrum., vol. 82, no. 2, 2011.

[8] A. Shymanska, "Effect of high-efficiency emitter on noise characteristics of electron amplifiers," J. Comput. Electron., vol. 14, no. 1, pp. 341-351, 2015.

[9] M. A. Furman and M. T. F. Pivi, "Probabilistic model for the simulation of secondary electron emission," Phys. Rev. Spec. Top. Accel. Beams, vol. 5, no. 12, pp. 82-99, 2002.

[10] N. W. S.Frey, J. Löffler, C. Ballif, "Characterization of Amorphous Silicon Based Microchannel Plates with High Aspect Ratio," IEEE Nucl. Sci. Symp. Conf. Rec., no. This Issue.

[11] T. Koshikawa and R. Shimizu, "A Monte Carlo calculation of low- 
energy secondary electron emission from metals," J. Phys. D. Appl.

Phys., vol. 7, no. 9, pp. 1303-1315, 1974.

[12] M. Belhaj, T. Tondu, V. Inguimbert, P. Barroy, F. Silva, and A.

Gicquel, "The effects of incident electron current density and

temperature on the total electron emission yield of polycrystalline

CVD diamond," J. Phys. D. Appl. Phys., vol. 43, no. 13, 2010.

[13] M. Belhaj, T. Tondu, V. Inguimbert, and J. P. Chardon, "A Kelvin probe based method for measuring the electron emission yield of insulators and insulated conductors subjected to electron irradiation," J. Phys. D. Appl. Phys., vol. 42, no. 10, 2009.

[14] K. Kanaya, S. Ono, and F. Ishigaki, "Secondary electron emission from insulators," J. Phys. D. Appl. Phys., vol. 11, no. 17, pp. 2425 2437, Dec. 1978

[15] H. Seiler, "Secondary electron emission in the scanning electron microscope," J. Appl. Phys., vol. 54, no. 11, 1983.

[16] R. Cimino et al., "Can low-energy electrons affect high-energy physics accelerators?," Phys. Rev. Lett., vol. 93, no. 1, pp. 014801$1,2004$.

[17] S. F. Mao, Y. G. Li, R. G. Zeng, and Z. J. Ding, "Electron inelastic scattering and secondary electron emission calculated without the single pole approximation," J. Appl. Phys., vol. 104, no. 11, 2008.

[18] E. Kieft and E. Bosch, "Refinement of Monte Carlo simulations of electron-specimen interaction in low-voltage SEM," J. Phys. D. Appl. Phys., vol. 41, no. 21, 2008.

[19] T. Verduin, "Quantum Noise Effects in e-Beam Lithography and Metrology," 2017.

[20] E. Schreiber and H. J. Fitting, "Monte Carlo simulation of secondary electron emission from the insulator $\mathrm{SiO} 2$, , J. Electron Spectros. Relat. Phenomena, vol. 124, no. 1, pp. 25-37, 2002.

[21] N. Bundaleski, M. Belhaj, T. Gineste, and O. M. N. D. Teodoro, "Calculation of the angular dependence of the total electron yield," Vacuum, vol. 122, pp. 255-259, 2015. 